\title{
Managing Social Media Content During a Global Crisis: Interview With Ben Read, Social Media Manager at World in Motion
}

\author{
Charles Mountifield and Stirling Sharpe \\ University of Canberra
}

In 2020, nations around the world quickly became engulfed in a crisis with the announcement that the COVID-19 virus had mushroomed to the point that it had become a pandemic. Subsequent to national government attempts to curtail the spread of the virus, there was an immediate impact on the sport industry. For sporting organizations, there was a cessation of competition and a lockdown for all those in the industry. In the absence of live sport, social media producers have been required to think outside of their normal content avenues to create posts that will engage their followers. Sport agency World in Motion-active in soccer, rugby, golf, boxing, and cricket - is an avid user of social media by way of engaging with clients and sports fans alike. As a result of the COVID-19 crisis, there have been significant changes to the focus of the company's social media output.

Ben Read, the organization's social media manager, is responsible for the design and dissemination of information through various platforms, notably, Twitter (8,715 followers) and Instagram (2,251 followers). Read has been employed at World in Motion since May 2019. Prior to this, Read was an account manager at Rockford Components and completed a psychology degree in 2017. From 2014 to 2017, while at university, Ben was also an event manager for the university student union, where he honed his social media skills. This interview provides background to the customary aspects of Read's role, including content creation and connecting with clients and sports fans. The interview then focuses on how Read's approach to social media has changed in light of the global crisis, based principally around the cessation of live sport and the company's wish to provide relevant and useful social media content.

Mountifield and Sharpe: What is World in Motion? What is your role? What do you do on a day-to-day basis?

Read: We are a global sports management agency operating across football, rugby, golf, boxing, and cricket. Some of our clients include Premier League and international footballers, international rugby players, and champion boxers from

The authors are with the University of Canberra, Bruce, ACT, Australia. Mountifield (charles .mountifield@canberra.edu.au) is corresponding author. 


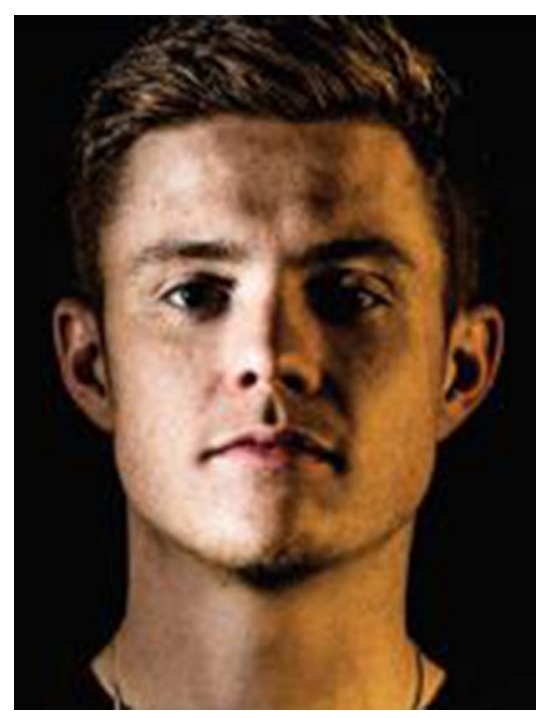

Ben Read - Social media manager at World in Motion.

all over the world. As social media and database manager, I am solely responsible for the online presence of the company through our various social media channels as well as our website. My main goal is to make sure that our athletes are promoted in a professional and engaging manner through our social media channels, highlighting their personal achievements and displaying the vast array of client talent that we have as an agency. I do this by creating our own unique video and image content, running campaigns and competitions, recording podcasts, and reposting relevant content from other sources. Other day-to-day tasks include the general management and maintenance of the channels, such as replying to direct messages and creating Instagram story highlights.

Mountifield and Sharpe: What platforms do you use, and how do you focus the message you want to convey? Is the process strategically oriented? How do you ensure that the branding and message are consistent?

Read: Our two main social media channels are Instagram and Twitter; however, just recently we created a "World in Motion TV" YouTube channel, which we have very exciting plans for. Due to the nature of the platforms themselves, we use our Instagram and Twitter channels slightly differently. We think of our Instagram page as a "shop window," where users can navigate to our page and instantly get an idea of who we are, what we do, and who we represent by means of short video clips, eye-catching graphics, and longer IGTV (Instagram TV) videos. On the other hand, our Twitter channel is used more as an update platform, especially on match/ event days. This is where users can get a deeper insight into World in Motion and our clients, posting client updates that range from a penalty save in League Two or a goal in the Premier League to round-by-round updates in championship boxing matches and action from the first tee of a golf tournament. 
Mountifield and Sharpe: An event such as COVID-19 has not been seen in the age of social media_-what are your initial thoughts on the impact of COVID-19 on social media's role?

Read: Despite the truly terrible global crisis, my thoughts on the impact of COVID19 on social media are mostly positive. I think the crisis has completely revolutionized the way people are using social media platforms, and a great deal of the content that has been created during this widespread lockdown period has been fantastic. It's almost forced creators into being super creative, as well as providing an incredibly powerful platform for campaigns to gain support. One that springs to mind is the recent campaign of Captain Tom Moore in the United Kingdom. The 99-year-old World War II veteran has (at the time of this writing) raised a staggering $£ 18$ million for National Health Service (NHS) charities in a number of days by walking one hundred lengths of his garden by his one-hundredth birthday. This, of course, wouldn't have been possible without social media, and Captain Tom's Twitter account at the time of this interview has 131,000 followers, gaining almost 15,000 followers a day since it was created. Another popular social media campaign in the United Kingdom at the moment is Joe Wicks' 9 a.m. workout; Joe is a health and fitness influencer who has set out to get the nation, especially children who are off from school, moving and active. His Instagram live videos have been getting millions of hits every day. Using those as prime examples, I think that, moving forward, this crisis is going to continue to improve the social media landscape, improving both the content that is being created and how we consume it to our benefit.

Mountifield and Sharpe: How do you engage with clients and other followers of your platforms? Is it one-way communication or do you interact with contributors?

Read: Yes, I often interact with contributors on our channels. Whether that is simply replying to a message, liking a comment, or retweeting a relevant tweet, I think it is important to have some kind of two-way communication. We also use our Instagram story to interact with our clients a lot. We wish each individual client a happy birthday with a personalized Instagram and Twitter graphic, and I am constantly reposting content on to our story from clients themselves or from their clubs.

Mountifield and Sharpe: In light of the current global health crisis, what changes have you made to the company's social media output?

Read: We have had to make some relatively big changes, to be honest. Naturally, that has had to happen as our main source of content has completely dried up, because there is no sport being played all over the world. Like many clubs, we have shifted some of our attention to reliving past glories by posting "On This Day" features. I really like what we've done with that, because it is not only a great way of filling the void in current sports content at the moment but it also really engages our clients and their fans by reminding them of more positive times, something that I think is very important in the current climate. Also, we have just embarked on the creation of a brand new online podcast with Ray Stubbs (former sports presenter and pundit) and Jonathan Davies OBE (former Welsh international rugby legend). At the time of this interview, we have just recorded our second episode, in which 
we had Premier League footballer and Wolverhampton Wanderers captain Conor Coady as a guest, and we have many more fantastic guests lined up for future episodes. We are really pushing this over our socials during this lockdown period, especially over on our new "World In Motion TV" YouTube channel, and it is something I am very excited about. Not only does this provide our organic followers with great content from some of the sporting world's biggest names, but it also engages audiences that are usually difficult to reach.

Mountifield and Sharpe: What changes have you observed in terms of general social media output in sport?

Read: I think the general social media output in sport has been similar to the path we have taken with ours. Most are attempting to rapidly adapt to the situation by creating content to fill the void left by live sport. There are a few ways that this has happened, and I think each strategy has differed depending on the target audience. Older audiences are more inclined to consume and enjoy content that is reminiscent of the past. Posting highlights and montages from memorable games or moments in a match is very popular at the moment and is flooding the timelines of sporting social media channels. There is also more insightful content that I think appeals to audiences that are in the middle bracket in terms of age. Some channels are trying to create content that gets their audience up close and personal with their favorite sports stars during the COVID-19 crisis, whether in the form of a podcast, video $\log$, or online interview. Also, there is a wave of content that is aimed at engaging the younger generation, who are incredibly receptive to online gaming and how this links to the real sporting world. EA Sports just rolled out their FIFA 20 \#StayHome Cup, in which professional footballers play virtually against each other, representing their actual teams.

Mountifield and Sharpe: What impact do you think your company's changes to social media - and those of others-are having?

Read: Not only do I think that we're providing quality content for sports fans during this testing time, but I see this changing the landscape of sports social media for good. I think channels are going to become more creative and we are going to see better quality content as a general rule, moving forward. Personally, I'm very excited to see the innovation in sports social media when the time comes for competitions to safely return. I'd like to think that the general impact of social media during this testing time can bring a bit of positivity and joy to sports fans across the world.

Mountifield and Sharpe: What sort of things are you looking for from your clients for a retweet and has this changed during the pandemic?

Read: Yes, I think naturally this has changed slightly during the pandemic, because there is some kind of social responsibility for sport stars who are in the public eye at this current moment. Some of our clients are heroes and role models in the eyes of their fans, and I think in times like these that responsibility can be used incredibly powerfully. Anything that is positive or spreads positive energy and is worth a retweet or post I will consider, because I think it's very important to highlight these things now. I know I have already mentioned Captain Tom Moore 
and his incredible effort to raise money for NHS charities, but this is a prime example of what is currently worth a retweet. Along with this, of course, you need to have sports-related content to post too. Anything positive coming out of certain clubs/leagues is also worth considering, as well as having a bit of fun with the numerous gaming competitions featuring sports stars that are happening.

Mountifield and Sharpe: How do you measure the success and relevance of social media? Do you track usage and other metrics?

Read: Yes, and I believe it's very important, because without it you are almost wandering in the dark in terms of posting your content! I try to measure as much as I can, as long as the metrics are relevant and offer an insight that I can use to our advantage. I track statistics from simple follower count to specific engagement on individual posts. One of the more interesting trends I have discovered through our channel is the way our audience engages with different forms of media. I have learned which posts require video or a graphic and which should go on our Instagram story, et cetera, in order to maximize our engagement. Tracking our Twitter metrics has also been very influential on the way I use our Twitter account. We have a few very active and high-profile clients that are popular on Twitter, and it is very powerful to use their dedicated following through retweeting and reposting. We have seen some of our posts rack up nearly 200,000 impressions through simple audience targeting via our clients' channels.

Mountifield and Sharpe: Has social media engagement increased across your managed accounts since COVID-19 forced competition suspensions, postponements, and cancellations?

Read: It has, yes. I think this is the case across all social media, to be honest! Unsurprisingly, people are consuming more media during the lockdown period, because they are confined to their homes. That may be through social media, TV, podcasts, and even radio. I think sports fans, in particular, are searching and consuming more content, because their favorite sporting competitions have been canceled or postponed. Sport is such an integral part of life for many, and one of the only ways for people to stay connected to their clubs, teams, and favorite players is through the consumption of social media. As we have seen already, I think it has really highlighted the power of social media and media in general if it is used in the correct manner.

Mountifield and Sharpe: Within the current COVID-19 crisis, how do you decide where to post content?

Read: It's very similar to before the crisis, to be totally honest. I think I have worked out which content works best on which platform for our audience, and that's what I'm continuing throughout the COVID-19 crisis in order to ensure that we can reach them with the best content we can. However, I will be experimenting a little in the weeks to come with the launch of our new YouTube channel with the hope of engaging and growing a whole new audience through that medium.

Mountifield and Sharpe: Going back to your new YouTube channel-how has COVID-19 influenced the timing of the launch and the content? 
Read: COVID-19 has influenced both the launch and the content of the YouTube channel massively. We have been discussing our strategy for launching a "World In Motion TV" concept for a while now; however, we never got to the point at which we had something concrete to start with. I think we were wary that to create something successful and worthwhile in an already very saturated market such as YouTube it needed a lot of attention and thought, and this was difficult in between very busy transfer windows and the action that comes with that! Having said this, we thought it was the perfect opportunity to start with the whole sporting world at a complete halt due to the pandemic. We pushed through a few ideas quite quickly and we've created a platform that I think we can build on in the weeks and months to come. As for the content, obviously I think the current crisis has influenced what we will be creating to start with; however, we have a great starting point with the creation of the podcast I previously mentioned. The plan moving forward is to do vodcasts, in which we not only record the audio from Jiffy \& Stubbsy podcast episodes but also have a video element, too, which will be put exclusively on our YouTube channel. This gives a completely different dimension from the podcast and allows our hosts and guests to interact with their fans on a new level. I know some of the sporting broadcast giants in the United Kingdom such as Sky Sports and BT Sport have been doing really well with these of late.

Mountifield and Sharpe: You say you expect "better quality content" to be on sport social media in the future-what do you mean by this?

Read: At the moment I think we are just seeing the start of a total creative reset on social media, especially in the sporting world. The pandemic is forcing creators and channel managers to get as creative as possible with very limited resources to work with. As we know, there are no games or competitions being played, no training sessions being carried out, no opportunity to go to grounds or stadiums to record content, and these are all the regular go-to methods when sporting channels want to create engaging content. With that not being available, it is almost forcing creators to think outside of the box, and if they don't, then their content streams are going to completely dry up, which nobody wants at a time when social media is incredibly active with people isolating in their homes. So having said this, I am really excited to see what the content is going to look like when things do finally return to some kind of normality. I think creators will be taking the creative methods that they have learned during this lockdown period and applying them to full effect when they have access to all of the previous sources of content (players, managers, games, competitions, et cetera).

Mountifield and Sharpe: For the clients whose social media you manage, how has COVID-19 changed your operations?

Read: I think this has been the most challenging part of the COVID-19 crisis in terms of social media work for me personally. It can be very difficult to help our clients interact with their fans when the main reason why they followed in the first place (sport) has been taken away. I think the main focus for me has been to really try to push our clients' individual personalities across, using this time to help them connect with their followers and fans in a way that wouldn't have been possible before. For example, we ran a competition on Andrew "Beef" Johnston's (top 
European Tour golfer) Instagram channel a few weeks back that did very well. We gave three of his followers the opportunity to win a "beer with Beef" and share an Instagram live video with Beef on a Saturday night. We were really happy with how this went, because it not only gave the competition winners the ultimate opportunity to connect with him by having a live conversation but it also gave the followers that were watching a chance to connect with Beef by hearing his stories/sending him questions via the live feed.

\section{Concluding Discussion}

The interview with Ben Read showcases the changes that one sport agency has made in response to the COVID-19 pandemic. Read's experience and observations indicate that sport content on social media will need to continue to evolve post-pandemic and that consumers' long-term expectations may be different. Social media producers were invariably focused on creativity; however, with their most prominent source of content missing for several months, this requirement has been amplified. We agree with Read's position statement in that the "go-to" methods will evolve into "better quality content" in the future. However, as Read points out, a change in content does not negate the need for organizations to properly position their content across their platforms or to analyze the engagement statistics in order to understand their audiences. Recognizing the preferences of social media followers across all platforms and posting relevant content will remain an important objective for organizations. 